\title{
Hybrid Enriched Stacked Auto Encoder and FFO- MPARCNN Algorithm for the Multispectral Image LULC Classification
}

\section{Bhuvaneswari E}

Anna University Chennai

Anil Kumar T Ch

Vignan's Foundation for Science Technology and Research

Haqqani Arshad

Yanbu Industrial College

Dhanagopal $\mathbf{R}$

Chennai Institute of Technology

Bharath Kumar Narukullapati ( $\nabla$ nbk_eee@vignan.ac.in )

Vignan's Foundation for Science Technology and Research

\section{Research Article}

Keywords: Multi-spectral images, feature extraction, LULC classification, Modified Pre-Activation Residual Convolutional Neural Network and Stacked Auto Encoder, Hyper-parameters optimization

Posted Date: July 16th, 2021

DOI: https://doi.org/10.21203/rs.3.rs-717451/v1

License: (c) (1) This work is licensed under a Creative Commons Attribution 4.0 International License. Read Full License 


\title{
Hybrid Enriched Stacked Auto Encoder and FFO-MPARCNN Algorithm for the Multispectral Image
}

\section{LULC Classification}

Name : Dr. E. BHUVANESWARI

Affiliation: Dept. of CSE, Anna University

E-mail: bhuvaneswari.e.phd@gmail.com

Name : T Ch Anil Kumar

Affiliation: Dept. of Mechanical Engineering, Vignan's Foundation for Science Technology and Research, Guntur, India

E-mail: tcak_mech@vignan.ac.in

\section{Name : Haqqani Arshad}

Affiliation: Dept. of Mechanical Engineering, Yanbu Industrial College, Yanbu, Saudi Arabia

E-mail: $\underline{\text { arshadh@rcyci.edu.sa }}$

\section{Name: Dr.R.Dhanagopal}

Affiliation: Director, Centre for System Design,Chennai Institute of Technology, Chennai , Tamil Nadu, India

E-mail: dhanagopalr@citchennai.net

(corresponding author)

\section{Name : Bharath Kumar Narukullapati}

Affiliation: Dept. of Electrical and Electronics Engineering, Vignan's Foundation for Science Technology and Research, Guntur, India

E-mail: nbk_eee@vignan.ac.in

\begin{abstract}
The satellite imagery classification task is fundamental to spatial knowledge discovery. Land Cover and Land usage (LULC) maps are created using a variety of image classification techniques, making it easier to conduct research on spatial and ecological processes as well as human activities. One of the most well-known applications of geographical monitoring is LULC classification. Owing to its improved feature learning and feature expression capacity, the convolutional neural network (CNN) has made several breakthroughs in feature extraction as well as classification of multispectral images in recent times as compared with conventional machine learning approaches. But on the other hand, standard CNN models have certain disadvantages, for instance, a large number of layers, which contribute to difficult computing costs. The Hybrid Enriched Stacked Auto Encoder and Pre-Activated Residual Convolutional Neural Network combined with a Fruit Fly
\end{abstract}


Optimization Algorithm (HESAE-FFO-MPARCNN) has formulated where FFO used to optimise parameters and thus enhance the accuracy of classification in this work to tackle this issue. The designed FFO-MPARCNN model with its modified hyperparameters produces higher classical models as PB-RNN, ResNet and FHS-DBN for computational efficiency and accuracy of classification.

Keywords: Multi-spectral images, feature extraction, LULC classification, Modified Pre-Activation Residual Convolutional Neural Network and Stacked Auto Encoder, Hyper-parameters optimization.

\section{Introduction}

One of the most important techniques in the quantitative analysis of remotely sensed images is multispectral image classification [1]. A pixel's characteristics are normally reported over a variety of spectral channels in multispectral images. The ground cover is typically mapped from remotely sensed data using a supervised image classification system. The relevant subfield of land cover monitoring is LULC classification. LULC refers to the method of categorising remote sensing images hooked on different land cover groups, for example, plantations, water, built-up areas, roads, and forests [2]. To identify satellite images or generate accurate maps from satellite data, Machine Learning (ML) algorithms for example k-means clustering, as well as maximum likelihood classifier [3], historically used. That being said, over the last decade, consumption of such deep learning-based methodologies to resolve domain-specific difficulties has grown in popularity also effectiveness [4]. Particularly in recent days, researchers utilized the Deep convolutional neural networks (DCNN) especially for computer vision tasks [5] besides are well suited for computer revelation problems for instance semantic segmentation of images, as they can acquire low-level as well as high-level features in a hierarchical fashion.

Many LULC classification studies have been reported in recent years utilizing multi-spectral LiDAR data [8]. Higher point density has been reached with the advent of LiDAR technology, implying a heavier numerical workload as the LULCclassification is done. While the above approaches have provided core land-based classificatory technologies for LiDAR data, those that are already researching with standard low-level LiDAR for example instability, entropy, and skewness metrics unable to obtain detailed high-level characteristics LiDAR data. Nowadays, deep learning strategies emphasis on network architecture layer depth as well as decrease the fittings of every other layer may draw in deep, high-level features from the original data which lead to enhanced accuracy and consistency in classification [9]. Credibility for image processing in conventional deep learning architectures has been developed for the convolutional neural network (CNN). 
From instruction to forecasting, CNN, a neuroscience-inspired multi-layered, deep learning framework, features a separate neural network. CNN learns spectral and spatial information from images that use stacked convolution kernels to obtain abstract characteristics of a high level. As even the neural network goes deeper, the CNN performing classification responsibilities through founding a relationship among input image samples and output labels. In many other vision-related tasks which including Visual Detection [11], scene labelling [12], including face recognition [13], because of the obtainability of a large amount of training data with the usage of effective enactment workplaces. The CNN model outperformed many standard neural network approaches (namely auto encoding, Sparse coding, restricted Boltzmann machine).

Throughout the model training stage, non-hyper-parameters are constantly modified which are dimension, input distance, number of convolution kernels, convolution kernel size, learning rate and pooling window size, which have a huge effect on the training and final projections. As a result, constructing a powerful CNN model and determining the necessary parameters for LULC classification responsibilities is extremely difficult and timeconsuming. That being said, there are no simple guidelines for optimising CNN hyper-parameters at the moment, and they are often decided by a designer's knowledge and instincts [15].

The Hybrid Enriched Stacked Auto Encoder and Modified Pre-Activation Residual Convolutional Neural Network with Fruit Fly Optimization Algorithm (HESAE-FFO-MPARCNN) are proposed in this work to reduce the computational complexity in the multi-spectral image-based LULC classification mission. In brief, the following are the key contributions of the proposed multi-spectral LULC classification design:

- $\quad$ Aimed at the multispectral land cover classification challenge, the HESAE-FFO-MPARCNN model was developed.

- The hyper-parameters of the built ESAE-MPARCNN model are addressed as well as configured using the FFO approach to deliver instructions for multi-spectral LULC classification.

Below is a description of the paper's structure: Work on machine learning and especially deep learning-based methods for classification in general, and LULC mapping and interpretation in particular, is discussed in Section. 2. The HESAE-FFO-MPARCNN model for multi-spectral sentimental-2 image-based land cover classification is defined in Section 3. The results of the experiment are illustrated and explained in section 4. Finally, Section 5 contains the closing remarks.

\section{Related Work}


Through deep learning outperforming traditional machine learning methods in classifying pictures, the remote sensing group has recently given the increased interest in using these techniques to identify LULC using multispectral and hyperspectral images (HSI). A modern patch-based RNN which is denoted as PB-RNN framework proposed and optimised for multi-temporal remote sensing data by integrating and then using the full multi-spectral, multitemporal, as well as spatial information in remote sensing images besides recognising them receive spatial and sequential interdependence [16].

In [17], a recurrent residual network (Re-ResNet) architecture was introduced that can acquire a joint spectralspatial-temporal feature exemplification surrounded by a centralized context. A residual convolutional neural network (ResNet) and a recurrent neural network (RNN) are merged into a single endwise architecture to achieve this goal. In [18] suggested a novel framework for multispectral and panchromatic image recognition using adaptive multi-scale convolutional neural networks and a perceptual loss feature.

In [19] proposed a deep learning-based multi-spectral LULC classification system. A spectral-texture classification model is built using the contourlet transform's excellent detail capture ability to acquire possible details to complement the spectral feature space, coupled with deep learning for feature selection and feature extraction. In [20] focuses on land use classification and suggests the Firefly Harmony Search (FHS) optimization algorithm for training the Deep Belief Neural Network (DBN).

To identify the HSI, [21] used a hybrid stacked autoencoder (SAE) architecture and support vector machine (SVM) classifier. The algorithm is modified based on a convolutional neural network in [21], then tests are conducted on multi-source remote sensing images of various geomorphologies occupied further down three separate climatic conditions to validate the Improved convolutional neural network's efficacy and scalability.

Concentrating on the classification of LULC from multispectral and hyperspectral images, this paper offers a state-of-the-art analysis by incorporating several different approaches documented in the literature into a standardized deep learning system that addresses various aspects of the issue. The power-scale and energy conservation, on the other hand, is a key concern for onboard processes. As a consequence, reducing the complexity of the models is an important concern for future work.

\section{Proposed Methodology}

The feasibility of a HESAE-FFO-MPARCNN was investigated in this study. In ESAE-DNN, three hidden layers are deployed at the softmax classifier for feature extraction, followed by classification with FFO- 
MPARCNN and hyperparameter optimization with FFO. After which, by using Intersection over Union (IoU), consistency, precision, recall, and f-measure assessment metrics, compare the results of HESAE-FFOMPARCNN, PB-RNN, ResNet, and FHS-DBN on multispectral image classification. The experimental result shows that the HESAE-FFO-MPARCNN model outperforms the baseline PB-RNN, ResNet, and FHS-DBN models for multi-spectral image LULC classification tasks.

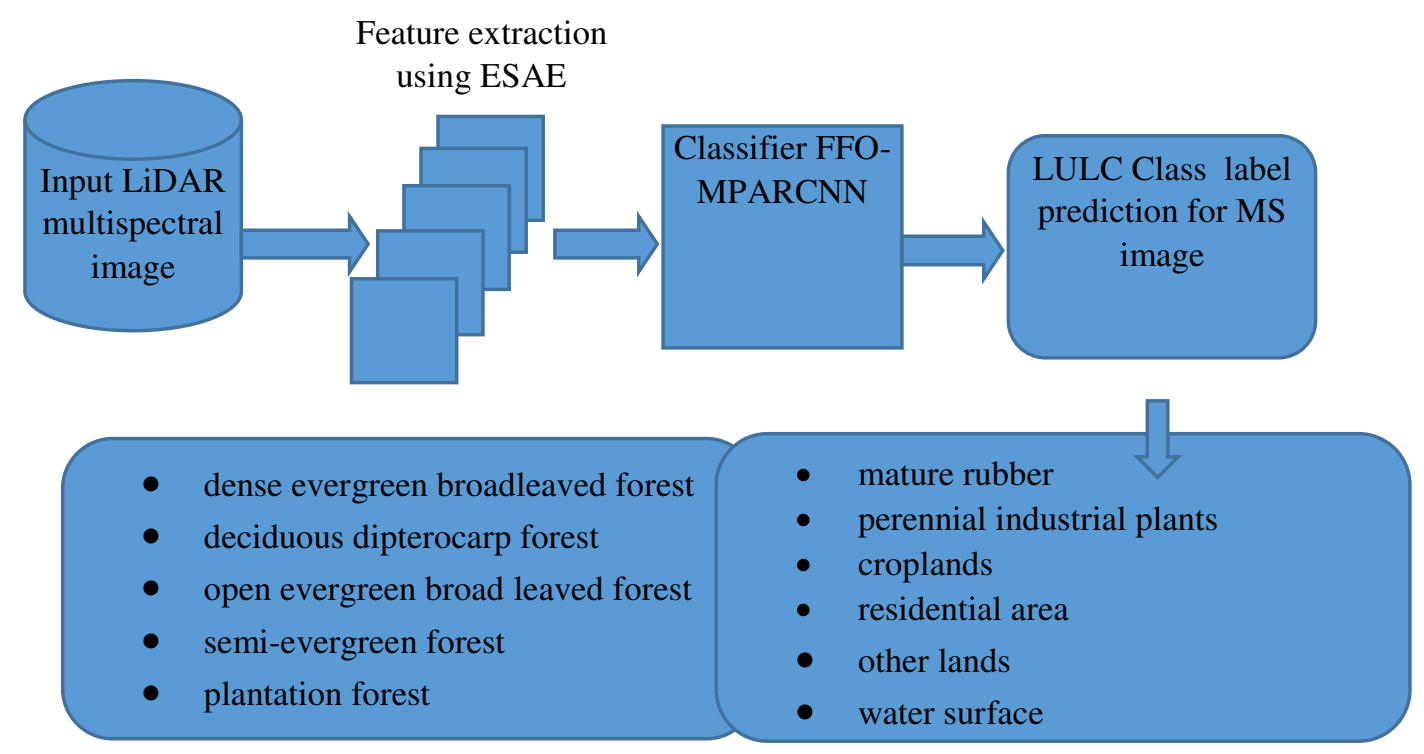

Fig.1. Multi-spectral LULC image classification using the proposed method

\subsection{Study Area}

The research was carried out in Vietnam's Dak Nong Province, in the Central Highlands (Figure 2). The climate in the province is humid tropical highland, and it is influenced by the dry, hot southwest monsoons. The research area covers $6516 \mathrm{~km} 2$ and is marked by significant disintegration, rendering LULC classification especially difficult. The natural forest is made up of areas of natural evergreen broadleaved, deciduous dipterocarp, mixed bamboo, including semi-deciduous forest with varying degrees of commotion, much of which is caused by humans [23]. 


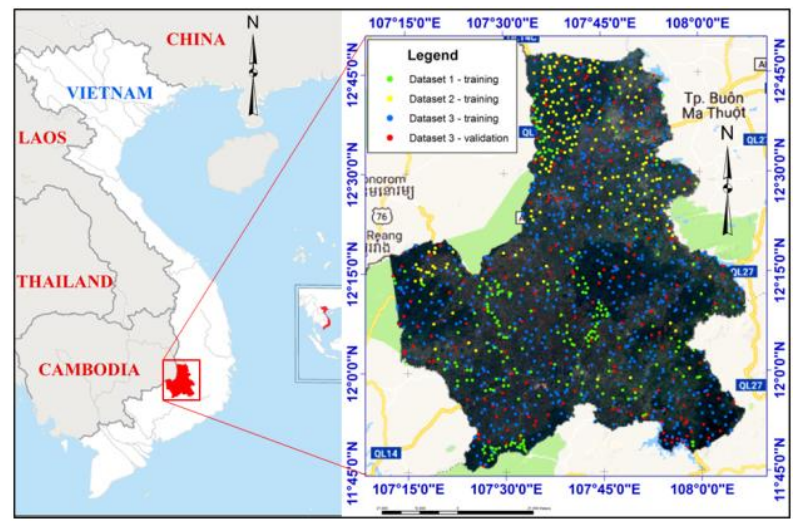

Fig. 2. The study area in Dak Nong province, Vietnam, with sample unit locations [23]

\subsection{Feature extraction by using ESAE-DNN}

This work proposes a model for LULC classification based on the DNN based feature extraction by using Enriched Stacked Auto-Encoders (ESAE-DNN) because this work has been greatly enhanced by the useful features and qualities of the deep networks to improve each classification problem evaluation metric. In combination with three hidden layer attribute extraction procedures, this same enriched stacked auto-encoder and the softmax layer have been developed for the construction of the LiDAR DNN classifier. The softmax layer is added to the last secret layer to carry out the classification task. The probabilities for the supplied record are given by the output layer for the class label generation of LULC.

Suppose that the ESAE network consists of three layers where the MS input is marked by MSI, image reconstitution is marked by $M S I^{\prime}$, and weight terms are given by $\omega^{(l, 1)}{ }_{,} \omega^{l(l, 1)}$ and biasing terms are given by $b^{(l, 1)}, b^{r(l, 1)}$ and (where $\left.l=1,2,3\right)$. As defined in Eq. (1), the input image on SAE maps was initially hidden $h(l)$.

$h_{i}^{l}=f\left(M S I_{i}^{l}\right)$

$f\left(I_{i}^{l}\right)=s_{f}\left(\omega^{(l, 1)} I_{i}^{(l)}+b^{(l, 1)}\right)$

The latent representation $h(l)$ then, as described in Eq. (2), was mapped back to $\Re$ from the corrupt version $I^{\prime}$

$r=g\left(h_{i}^{l}\right)$

$g\left(M S I^{l}\right)=s_{g}\left(\omega^{r(l, 1)} h^{l}+b^{l(l, 1)}\right)$ 
The $s_{f}$ and $s_{g}$ is known as a nonlinear activation function. The error in restoration $\Re E\left(I^{t}, \Re\right)$ reflects the costfunction of the SAE between the initial inputs $M S I^{\prime}$ and the rebuilding $\Re$.

$I_{S A E}=\sum_{l=1,2,3} \Re E\left(M S I^{\prime l}, g\left(f\left(I_{i}^{l}\right)\right)\right)$

$\Re e\left(M S I^{\prime}, \Re\right)=\left\|M S I^{\prime}-\Re\right\|^{2}$ the function of the functionalities. Also, SAE's secret units added a word $\rho$ for sparsity limitations, with additional penalty words $M S I$ being given for the objective feature in Eq. (4). The current cost feature of MSAE can then be updated as,

$I_{M S A E}(\theta)=J_{S A E}+\chi \sum_{j=1 \text { to } m} K L\left(\rho \| \rho_{j}\right)$

The cross-entropy within $\mathrm{c} \rho$ and $K \rho_{j}$ signifies the additional consequence terms Kullback-Leibler divergence (KL) expressed in Eq. (5).

$K L\left(c \rho \| K \rho_{j}\right)=\sum_{j=1}^{m} c \rho \log \frac{c \rho}{K \rho_{j}}+(1-\rho) \log \frac{1-c \rho}{1-K \rho_{j}}, K \rho_{j}=\frac{1}{k} \sum_{i=1}^{k} h_{i}$

In which the target amount of sparsity for the $j$ th unit is $K \rho_{j}$; the average rate of activation is $K \rho_{j}$; the number of cached units is $m$ and the weight of the terms of penalty is $\chi$. The input data for which the sparsity dictionary code is used in all layers for obtaining the optimum image character is displayed by defining a set of overcomplete base vectors. Centred on the sparse feature representation at the pixel level, three hidden layer sparse coding approaches [24] reveal the critical feature of an image. The deep network classification can be created by cascading a stacked auto-encoder using the softmax classification to allow the stacked autoencoder to contain 2 or more layers of auto-encoding. Three auto-encoders in the DNN classifier with ESAE are seen in fig. 3. Take as an input $\left\{I_{1}, \ldots, I_{n}\right\}$, variables as $\left\{Y_{1}, \ldots, Y_{n}\right\}$ and the corresponding output class the input vector fed through MPARCNN. The training approach aims to adjust DNN parameters for learning input vectors and classifying respective output value in addition to maximum precision, as defined input vectors for training. Training approach The following steps describe the ESAE-DNN classifier training process of feature extraction.

- The initial input vector $\left\{I_{1}, \ldots, I_{n}\right\}$ with the equivalent target, the vector is trained first on the first autoencoder layer. The input reconstruction attempts of the layer include $\left\{c_{(1,1)}, \ldots, c_{(1, c)}\right\}$ derivative characteristics with the autoencoder structure. 
- The second training autoencoder layer will be performed with the input vector, generating output vector $\left\{c_{(1,1)}, \ldots, c_{(1, c)}\right\}$ as input and generating output vectors $\left\{c_{(2,1)}, \ldots, c_{(2, R)}\right\}$. The output layer preparation is performed with the output vector information. The second layer of autoencoders to rebuild $c_{(1, C)}, \forall i=1,2, \ldots, C$.

- The final layer of an autonomic encoder is trained as the production vector for the second autonomic layer $\left\{c_{(2,1)}, \ldots, c_{(2, R)}\right\}$ and creates the output vector $\left\{c_{(3,1)}, \ldots, c_{(3, U)}\right\}$. The third layer is trained to accept an output vector as a backup. The third layer of input autoencoding efforts is the reconstruction $c_{(2, i)} ; i=1,2, \ldots, R$.

- A softmax classifier is cascaded in the autoencoder that is stacked. This layer is trained by consideration, $c_{(3, i)} ; i=1,2, \ldots, U$ as input vector, and $\left\{Y_{(1)}, \ldots, Y_{(N)}\right\}$ as target vector from training results, of the performance of the third autoencoder layer.

- Backpropagation is essentially used to boost the efficacy of the DNN as far as feature extraction is concerned. This fine-tuning is performed in a controlled manner by retracting the network along with the training results. MPARCNN is used to identify the characteristics after processing and feature extraction.

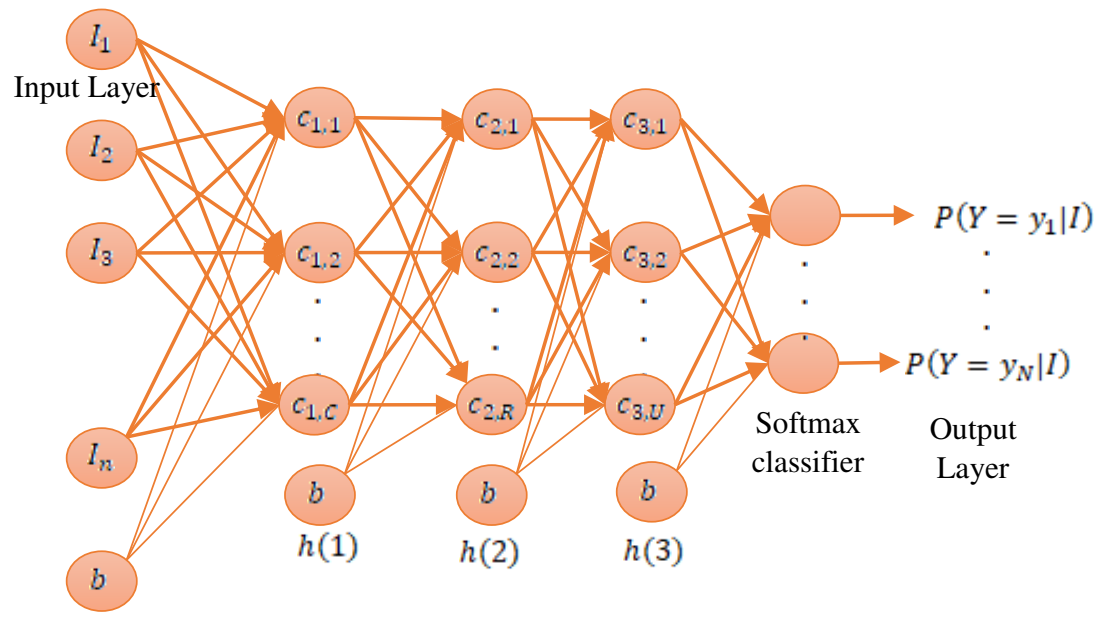

Fig.3. The architecture diagram of Feature extraction using the ESAE-DNN model

\subsection{Modified Pre-Activation Residual Convolutional Neural Network (MPARCNN)}

In comparison to related types of deep learning models, the MPARCNN approach tends dramatically to improve the performance of the recognition process with equal or less computational metrics. The MPARCNN approach proposed adopts the residual block as the key component in this work, in addition to that, there are two 
convolutional layers embedded in the residual block. This skip link also adds low and high-level functions. The deep network will thus minimise the issue of the gradient disappearing or explosion. Each residual estimate may be defined as

$O(x)=a f(R F(x)+x)$

In this case, $\mathrm{x}$ and $O\left({ }^{\circ}\right)$ is the residual block input images features and output of the class label. A residual study function is indicated by $R F$; the coevolutionary layer output is defined by $R F(x)$ before summation operation, and $f$ is indicated for the activation function. The batch standardisation (BN) and pre-activation mechanism in the suggested residual network block have been applied for optimum output in this area. Pre-activation architecture implementation is carried out utilizing the activation feature moving BN and Revised Linear Units (ReLU). The pre-activation residual block is expressed as

$O(x)=R F(x)+x$

The activation function af is ReLU and is defined accordingly.

$a f(x)=\max (0, x)$

If the signal is negative, Rectified Linear Unit (ReLU) forcefully converts the signal into 0, which may result in a loss of a few usable residual properties from the standard residual block. The images can be closely distributed in two units by identity mapping to ensure that features acquired from residual learning functions avoid failure. The network training process is thus streamlined through the pre-activation mechanism in addition to the output of the network generalisation.

The MPARCNN block, which included residues units, startup units and RCL, is considered to be an important element of this proposed architecture. The architectural phase can be summarised as follows, I the inputs are fed into the input layer and, if RCLs are implemented, ii) the IRRCNN [25] block input is supplied with the inputoutput of the initial units. The recurrent converter processes are performed in keeping with dissimilar sized kernels in the initial division. The preceding steps are applied with the output at the current time level as the configuration inside the convolution layer is recurrent. The outputs are then considered as an input for the following time stage at present. Similar processes are performed regarding estimated time stages.

The optimum detection precision guarantees stable functionality through a similar number of network parameters. RCL operations, as well as articulating based on RCN, were performed in line with the discrete-time 
steps. Suppose, the input sample of the $x l$ in the $l$ th layer of the MPARCNN block as well as the pixel of the $(i, j)$ sample of the ingredients on the kth map of the RCL functionality. Also, take into consideration that $O_{i j k}^{l}(t)$ output network is phase $t$ at that time. Then, the output is formulated as

$O_{i j k}^{l}(t)=\left(w_{k}^{f}\right)^{T} * x_{l}^{f(i, j)}(t)+\left(w_{k}^{r}\right)^{T}(t-1)+b_{k}$

In this case, $x_{l}^{f(i, j)}$ and $l^{\text {th }}$ federated RCL $\left(w_{k}^{r}\right)^{T}(t-1)$, as data, from the regular convolution layers. The $\mathrm{w}$ $w_{k}^{f}$ and $w_{k}^{r}$ values were correspondingly considered the usual convolutional layer as well as RCL of the $\mathrm{k}^{\text {th }}$ map function. Instead, $b k$ is represented as bias.

$y=f\left(o_{i j k}^{l}(t)\right)=\max \left(0, O_{i j k}^{l}(t)\right)$

In the equation above, the standard activation function for the ReLU indicates $f$. In the subsequent research, in which $y_{1 x 1}(x), y_{3 x 3}(x)$, and $y_{1 x 1}^{p}(x)$ represent the $y$ outputs of initiation units for dissimilar size kernels as well as average pooling stages. And, $\mathcal{F}\left(x_{l}, w_{l}\right)$ is the formula that can be determined as follows for the final effects of MPARCNN units;

$\mathcal{F}\left(x_{l}, w_{l}\right)=y_{1 x 1}(x) \odot \mathrm{y}(\mathrm{x}) \odot y_{1 x 1}^{p}(x)$

Where $\odot$ stands for the feature map axis concatenation process. In addition to the MPARCNN block inputs, RCNN unit outputs are added. The following equation indicates the residual activity of the MPARCNN block.

$x_{l+1}=x_{l}+\mathcal{F}(x l, w l)$

In this case, the next transformation block inputs are defined as $x_{l+1}, x_{l}$ is the input samples of MPARCNN block, $w l$ is the kernel weights of the $l$ th MPARCNN block and $\mathcal{F}\left(x_{l}, w_{l}\right)$ is a denote for outputs in the 1 of the MPARCNN layer. The numbers of maps are equivalent to the sizes of function maps for residual units as represented in Fig.4 in the MPARCNN block. Finally, with batch standardisation, MPARCNN block outputs are added.

Just $1 \times 1$ also $3 \times 3$ convolution filters were used during this execution, stimulated by IRRCNN [25] model. Furthermore, the minimum amount of network parameters is preserved. The non-linearity of the decision function can be reduced to influence the convolution layer by applying a $1 \times 1$ filter. As this is unchanged in IRRCNN blocks, in addition to nonlinearity is applied to the RELU activation functions, the input and output feature sized is essentially an undeviating prognostication of the same dimension. The dropout is 0.5 used 
subsequently any convolution layer in the transformation block. In the end, Softmax operation can be welldefined for the following ith classes for input features of MS sample $x$, weight vector W and L distinct linear functions:

$P(y=i \mid x)=\frac{e^{x^{T}} w_{i}}{\sum_{k=1}^{K} e^{x^{T}} w_{L}}$

A series of tests on several benchmark datasets were performed and results are compared to different models to test the proposed MPARCNN structure. Fig.5 illustrates the structure of the proposed MPARCNN.

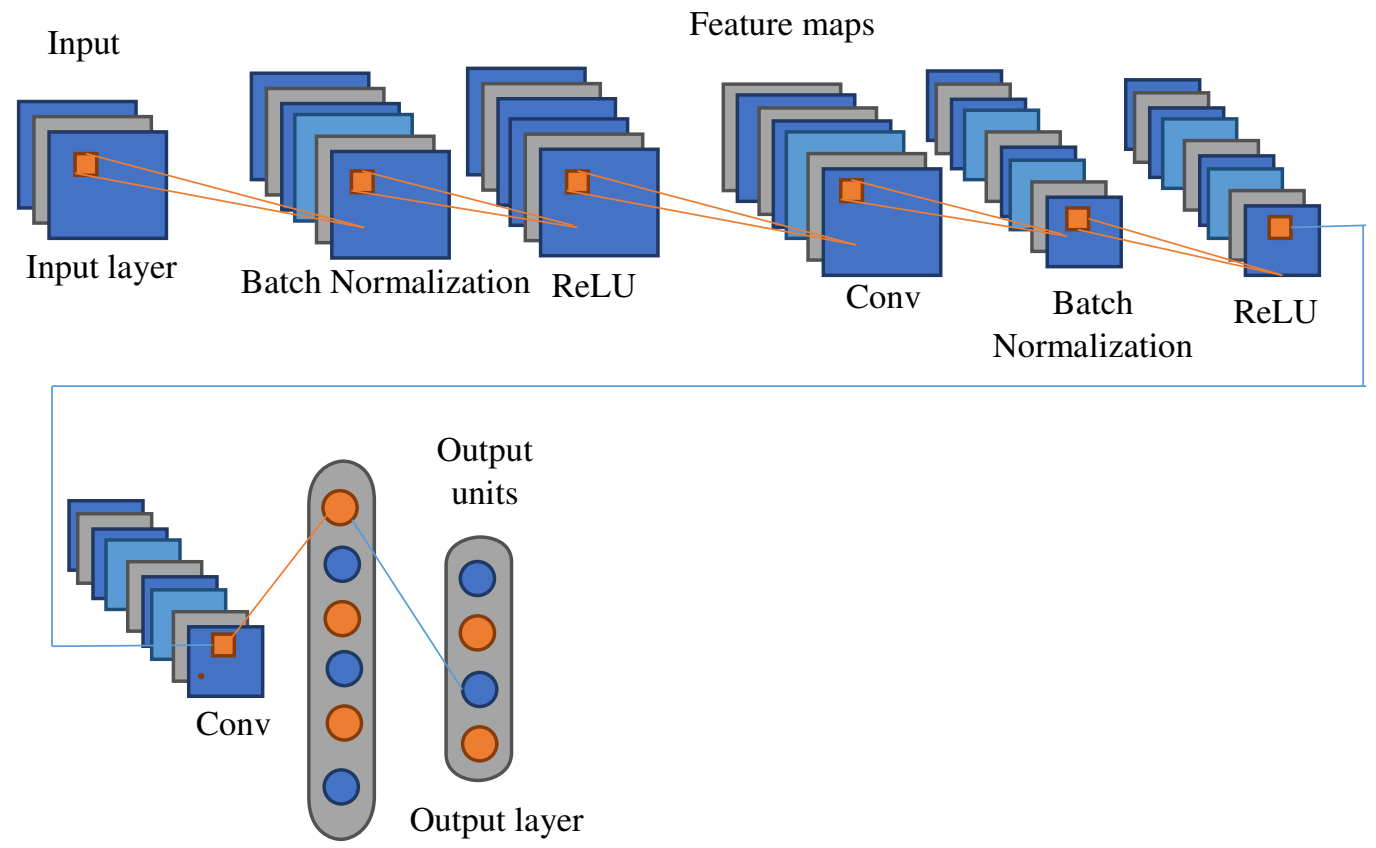

Softmax layer

Fig.4. The framework of the MPARCNN method

Objective Function of FFO: The network's weight is designed for error rate minimization, resulting in increased LULC classification accuracy. The weight values are tuned at each iteration, and the network is then trained.

$$
M S E_{i}=\min \left(\frac{\sum_{i=1}^{N}\left(D_{i}-P_{i}\right)^{2}}{N}\right)
$$

$D_{i}$ stands for Desired value, $P_{i}$ for Predicted value, and $\mathrm{N}$ for the Number of images.

\subsection{The Proposed FFO-MPARCNN Classifier}


The fundamental concept behind FFO-MPARCNN is to use FFO to identify the right MPARCNN parameters. The parameters are represented by the location coordinates of the fruit fly. A scent concentration judgement feature is present in all fruit flies. Classification precision is measured as a fitness function in FFO-MPARCNN. The MPARCNN candidate parameters are treated as flies' coordinates. As a consequence, all of the parameters should not be too great or too lesser, as well as should just look for the most acceptable parameter within a defined range. However, the relationship is unlikely to be a straightforward mapping relationship, since several extreme points contribute to increased complexity. According to the findings of the above study, the parameter selection of the MPARCNN problem is solved in this phase. Another is that the most appropriate parameters can be identified by searching only in a small area. The additional choice is to go to multiple extremes. FFO is a master at solving problems involving the two characters. FFO first initialises the scent with a small collection of random numbers, then chooses the most relevant MPARCNN parameters from the random numbers based on MPARCNN's classification precision and reports the parameters.

The random numbers will then be modified for the most appropriate parameters, as well as the adjusted number, along with an additional set of random numbers, which would be considered new-fangled candidates for the more appropriate parameters. FFO continues to pick the most appropriate MPARCNN criteria from new applicants based on MPARCNN's classification accuracy. If better parameters are discovered, FFO saves the data, adjusts the candidate parameters to the better parameters, then contemplates the adapted number plus an innovative set of random numbers to be the up-to-date aspirant parameters. Else, FFO will proceed by adding a fresh group of random numbers to the prehistoric random numbers rather than the modified ones. It is easy to see from the above definition that FFO propels the primary random parameters end to end a path towards the furthermost appropriate parameter instead of selecting them randomly. Here will use the optimal parameters determined by FFO to evaluate the model after MPARCNN. Have even let the fruit flies fly at random to help FFO discharge from the local optimum to boost the global searching ability. The FFO-MPARCNN steps are as follows:

Step 1: Set up the MPARCNN parameters, for instance, the number of layers, drop out rate per layer, units per layer, and L2 (or L1) regularisation parameters. Set FFO parameters like the swarm position radius, iteration number N, and group size $\mathrm{S}$ to their default values. Make ready the location of the fruit fly swarm at random.

Step 2: Using osphresis, give three-quarters of the fruit flies a random path and distance to look for food.

If $i<\left(\frac{1}{3}\right) S$ then $X_{i}=X_{\text {axis }}+$ Rand $Y_{i}=Y_{\text {axis }}+$ Rand 
Rand denoting random meaning.

Step 3: Subsequently the direction of the foodstuff cannot be determined, the distance to the origin is measured foremost $\left(D_{i}\right)$, followed by the scent concentration decision value (SC), which is the inverse of the distance.

$D_{i}=\sqrt{X_{i}^{2}+Y_{i}^{2}} ; S C_{i}=\frac{1}{D_{i}}, i=1,2, \ldots . N$

Step 4: To encounter the scent concentration $\left(\right.$ Smellconce $_{i}$ ) of the individual position of the fruit fly, substitute the SC into the smell concentration judgement feature.

Smellconce $_{i}=$ Function $\left(S C_{i}\right)$

Step 5: Classify the fruit fly with the largest attentiveness of odour in the swarm.

[bestSmell bestIndex $]=\max ($ Smell $)$

Step 6: Record the best scent attentiveness value as well as the $\mathrm{x}$, $\mathrm{y}$ synchronizes, and the fruit fly swarm can routine visualization to fly to that spot at this time.

Smellbest $=$ bestSmell

$X \_$axis $=X($ bestIndex $)$

$Y_{-}$axis $=Y($ bestIndex $)$

Step 7: Search to see if this process achieved the full number of iterations. Stop the iteration until finished.

Step 8: If not, increase the number of iterations by one, repeat Steps 2-5, and see if the scent concentration is healthier than the preceding iterative smell concentration; if not proceed to Step 6.

Step 9: A neighbour will be chosen at random in each iteration. If the selected neighbour has a lower MSE than the current state, the selected neighbour will be taken and its parameter values will be used as new parameter values, and eventually, the previous best SC value and $\mathrm{x}, \mathrm{y}$ coordinate will be obtained that are the values of the optimum parameters that must be discovered. After that, enter them into MPARCNN and proceed to the next level.

Step 10: MPARCNN training phase and evaluation of hyper-plane parameters using Eq (14). 
Step 11: MPARCNN testing phase, in which the class is assigned to a new data point using the judgement function from step 3 and distances are measured.

Step 12: Evaluate class using Eq.(19), i.e. the class is assigned to the pattern depending on the difference between the respective planes.

Step 13: Bring an end to the process. Understand the proposed FFO-MPARCNN algorithm's mechanism using this flow map, as seen in Fig.5.



Fig.5. Flowchart of FFO-MPARCNN

\section{Experimental results and Discussion}

To assess the performance of the built CNN for LULC classification based on a set of Sentinel-2 MSI scenes in the study area during the dry season of 2017 and 2018 with a total of 169 images were used. The tests were educated and tested on a computer with an Intel(R) Core(TM) i5-6500 processor running at $3.20 \mathrm{GHz}$ and 3.19 GHz, 16.0 GB of RAM, and an Intel(R) HD Graphics 530 graphics card. On multispectral image classification, the proposed FFO-MPARCNN is compared to PB-RNN, ResNet, and FHS-DBN using the Intersection over Union (IoU), accuracy, precision, recall, and f-measure. With a count value for each, the confusion matrix 
summarises the number of accurate and incorrect predictions. The error matrix yields some normal expressions, as follows: True positive (TP), True negative (TN), False Positive (FP), and False Negative (FN) are the four types of false positives and false negatives (FN). Both are the theoretical consequences of a single observation, with true positive (TP) and true negative (TN) being the right MS picture predictions. The following parameters for interpretation were determined using the measurements: accuracy, f-measure, recall, and precision.

Accuracy is specified as a percentage of the total number of accurate class mark predictions in LULC pictures. The approximation equation is as follows:

Accuracy $=\frac{T P+T N}{T P+F P+F N+T N}$

The recall is a percentage of the overall number of correctly labelled LULC image graphs that are denoted as positive values; it is often referred to as the true positive rate. The approximation equation is as follows:

Recall $=\frac{T P}{F N+T P}$

Precision refers to the percentage of expected positive values that are right in LULC classification. The approximation equation is as follows:

Precision $=\frac{T P}{T P+F P}$

F-measure is an important calculation of classifier efficiency for LULC class label generation since it provides the harmonic mean between precision and recall. The following equation is used to calculate it.

Fmeasure $=\frac{2 * \text { precision } * \text { recall }}{\text { precision }+ \text { recall }}$

IoU is defined as

$I o U=\frac{T P}{(T P+F N+F P)}$

\subsection{Precision Result comparison}




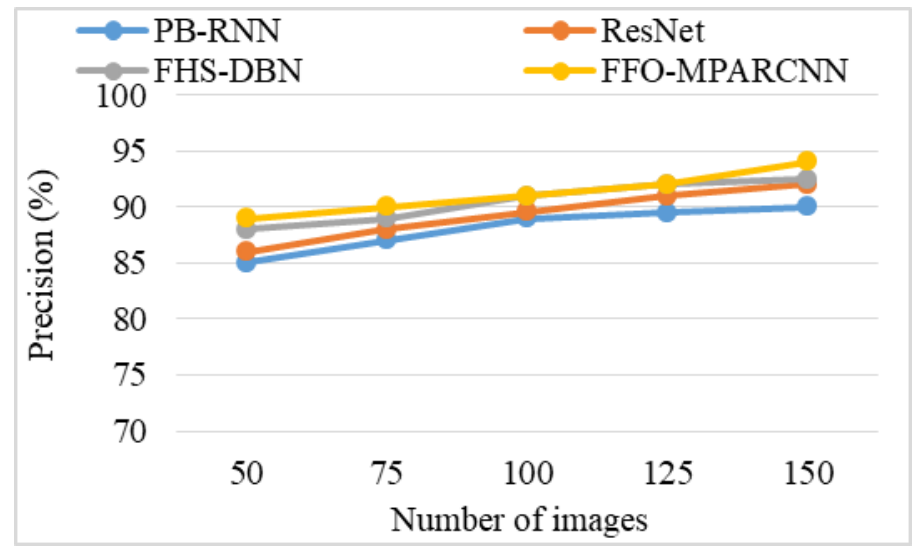

Fig.6. Precision performance comparison

The precision comparative results between PB-RNN, ResNet, FHS-DBN, and FFO-MPARCNN are seen in Figure 6. When compared to other approaches, the FFO-MPARCNN approach can achieve a high precision rate. It is a reliable method of obtaining classification data, with a precision rate of $94 \%$. When comparing the precision of current PB-RNN, ResNet and FHS-DBN have lower precision rates of 90\%, 92.5\%, and 92.55\%, respectively, than FFO-MPARCNN, since FFO will improve the generalisation potential of MPARCNN to increase classification precision. The numerical values of precision performance comparison are given in Table 1.

Table 1. The numerical values of precision performance comparison

\begin{tabular}{|l|l|l|l|l|}
\hline Input Image Data & PB-RNN & ResNet & FHS-DBN & FFO- \\
\hline 50 & 85 & 85.98 & 88 & MPARCNN \\
\hline 75 & 87 & 88 & 89 & 90 \\
\hline 100 & 89 & 89.6 & 91 & 91 \\
\hline 125 & 89.5 & 91 & 92 & 92 \\
\hline 150 & 90 & 92 & 92.5 & \\
\hline
\end{tabular}

\subsection{F-measure Result Comparison}




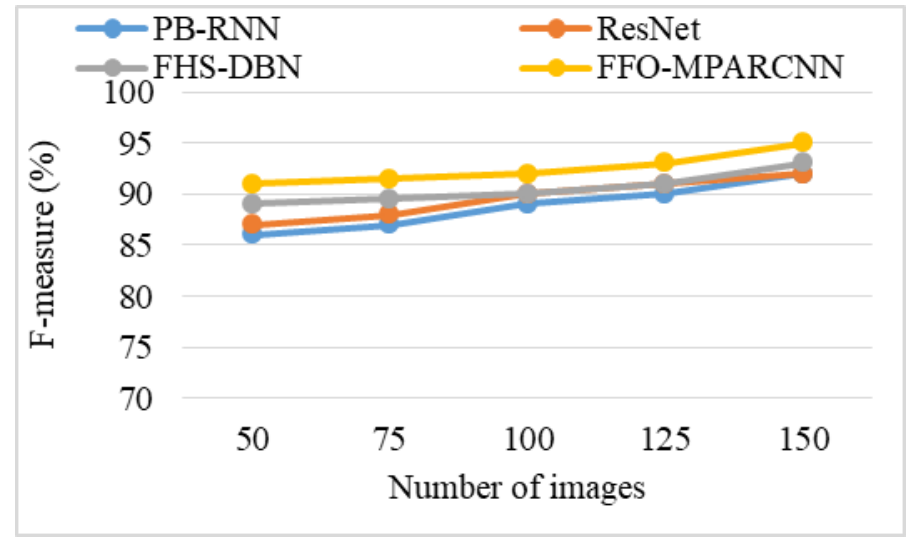

Fig.7. F-measure performance comparison

The F-measure contrast results between proposed PB-RNN, ResNet, FHS-DBN, and FFO-MPARCNN are seen in Fig.7. The suggested FFO-MPARCNN approach has a high F-measure value of 95\%. When compared to the current system PB-RNN, ResNet and FHS-DBN have lower grades of 92\%, 92\%, and 93\%, respectively, indicating that the proposed work will have improved RA grading performance. Given the additional information present in the additional spectral bands, FFO-stability MPARCNN's and high f-measure offer a potential solution to such a classification problem of MS images, and the additional information present in the additional spectral bands increases the distinction between land cover groups. The explanation for this is that a new technique for using FFO to develop the structure of MPARCNN has a range of advantages for neuroevolution, including the fact that it is conveniently parallelizable and scalable, and that it can be used as any tool for training neural networks, enhancing recall. The numerical values of the f-measure performance comparison are given in Table 2.

Table 2. The numerical values of f-measure performance comparison

\begin{tabular}{|l|l|l|l|l|}
\hline Input Image Data & PB-RNN & ResNet & FHS-DBN & FFO- \\
\hline 50 & & & & MPARCNN \\
\hline 75 & 86 & 87 & 89 & 91 \\
\hline 100 & 87 & 88 & 89.5 & 91.5 \\
\hline 125 & 89 & 90 & 90 & 92 \\
\hline
\end{tabular}




\subsection{Recall Result Comparison}

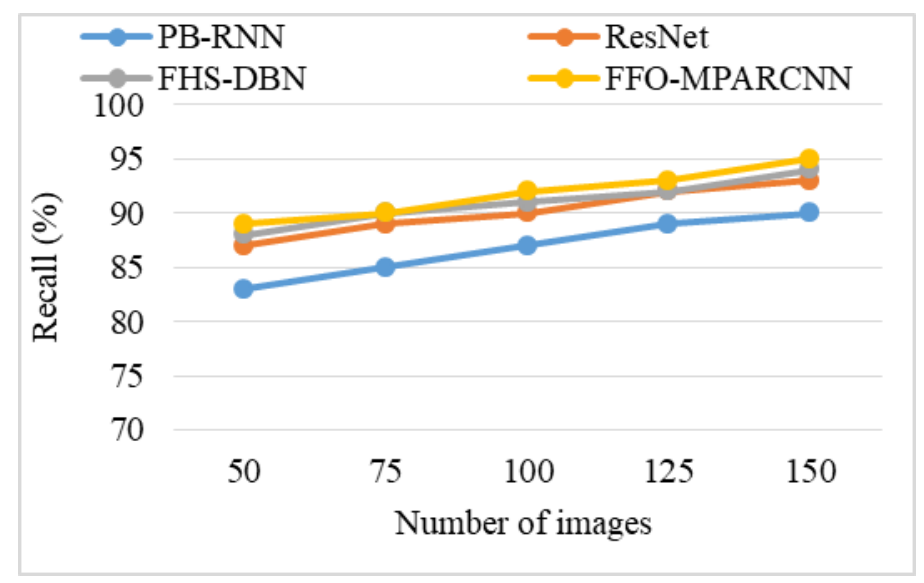

Fig.8. Recall performance comparison

The recall comparative findings between proposed PB-RNN, ResNet, FHS-DBN, and FFO-MPARCNN are seen in Fig.8. The suggested FFO-MPARCNN process has a high recall value of 95\%. It is clear from the findings that the proposed FFO-MPARCNN has a high recall rate value, suggesting a high cluster forming rate. When comparing the recall rates of the current systems, PB-RNN, ResNet, and FHS-DBN, the proposed work has a lower recall rate of $90 \%, 93 \%$, and $94 \%$, respectively, indicating that it can have better grading outcomes than the existing system. Most importantly, the use of an FFO and MPARCNN-based classifier in this study solved the issue of poor convergence and significantly reduced computing requirements for MS image classification. This demonstrates the potential utility of the proposed FFO-MPARCNN method in processing such high-dimensional imagery. The numerical values of recall performance comparison are given in Table 3.

Table 3. The numerical values of recall performance comparison

\begin{tabular}{|l|l|l|l|l|}
\hline Input Image Data & PB-RNN & ResNet & FHS-DBN & FFO- \\
\hline 50 & 83 & 87 & 88 & MPARCNN \\
\hline 75 & 85 & 89 & 90 & 90 \\
\hline
\end{tabular}




\begin{tabular}{|l|l|l|l|l|}
\hline 100 & 87 & 90 & 91 & 92 \\
\hline 125 & 89 & 92 & 92 & 93 \\
\hline 150 & 90 & 93 & 94 & 95 \\
\hline
\end{tabular}

\section{Accuracy comparison}

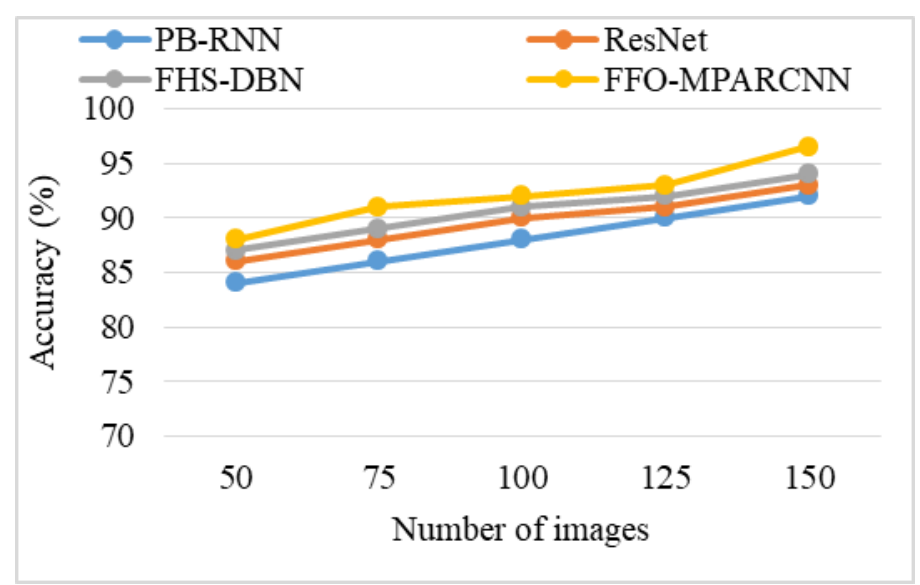

Fig. 9. Result of Accuracy

The accuracy relation for MS image classification is seen in the graph above (Fig. 9). As the amount of data is expanded in proportion to the accuracy value, the accuracy value increases linearly. From this table, it can be seen that the proposed FFO-MPARCNN effectively selects the cluster core for MS image classification with a high precision of $96.5 \%$. In terms of better classification performance for MS images with high accuracy levels, previous methods such as PB-RNN, ResNet, and FHS-DBN achieve low accuracy of 92\%, 93\%, and 94\%, respectively, compared to the FFO-MPARCNN. This approach can efficiently increase the accuracy of MS classification by integrating the advantages of ESAE, which can extract effective features from data, and MPARCNN, which can automatically detect the best mode appropriate for applicable data. Table 4 displays the numerical effects of the accuracy efficiency comparison. The numerical values of the accuracy performance comparison are given in Table 4.

Table 4. The numerical values of accuracy performance comparison

\begin{tabular}{|l|l|l|l|l|}
\hline Input Image Data & PB-RNN & FHS-DBN & FFO- \\
& & & MPARCNN \\
\hline
\end{tabular}




\begin{tabular}{|l|l|l|l|l|}
\hline 50 & 84 & 86 & 87 & 88 \\
\hline 75 & 86 & 88 & 89 & 91 \\
\hline 100 & 88 & 90 & 91 & 92 \\
\hline 125 & 90 & 91 & 92 & 93 \\
\hline 150 & 92 & 93 & 94 & 96.5 \\
\hline
\end{tabular}

\section{IoU comparison}

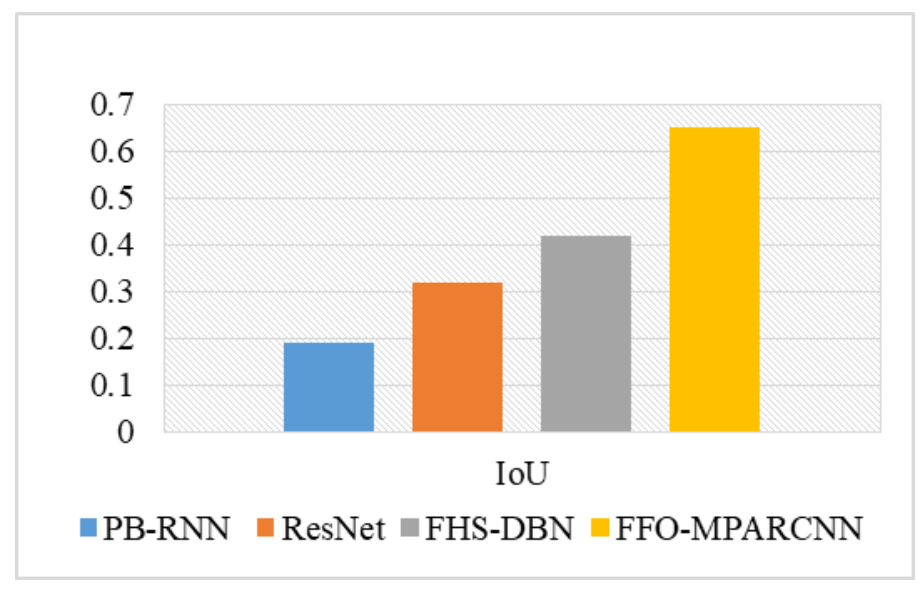

Fig. 10. Result of IoU

As seen in Fig.10, the PB-RNN classifier based on spectral indexes provides the lowest IoU results (0.19), suggesting that it is the least efficient strategy. ResNet produces $0.32 \mathrm{IoU}$ with equivalent preparation and testing as the FFO-MPARCNN; however, FHS-DBN produces 0.42 IoU, outperforming ResNet and the PBRNN model for MS classification. However, for the methods of PB-RNN, ResNet, and FHS-DBN, it is difficult to identify or find an acceptable threshold on class scores for classification with the specified number of validation samples. FFO-MPARCNN outperformed the PB-RNN, ResNet, and FHS-DBN with 0.65 IoU, which is higher than the PB-RNN, ResNet, and FHS-DBN. This highlights the importance of adaptive residual relations in enhancing the accuracy of MS classification models. Furthermore, despite using FFO to maximise the threshold and providing the best efficiency, the FFO-MPARCNN model shows that integrating FFO can provide a richer network representation, allowing for better classification outcomes. The numerical values of the IoU performance comparison are given in Table 5. 
Table 5. The numerical values of IoU performance comparison

\begin{tabular}{|l|l|l|l|l|}
\hline Methods & PB-RNN & ResNet & FHS-DBN & FFO- \\
\hline IoU & & & & MPARCNN \\
\hline
\end{tabular}

\section{Conclusion and Future Work}

This work proposed a CNN model for ground cover classification from multispectral satellite images with refined hyper-parameters. This study's major contributions can be summarised as follows: (1) The time complexity of the MPARCNN model is optimised by FFO, and (2) in the FFO-MPARCNN model, four hyperparameters remained examined to acquire the optimal values through the control variable method, namely number of layers, number of units at each layer, dropout rate at each layer, and L2 (or L1) regularisation parameters; using the optimal values of the hyper-parameters of MPARCNN by using the FFO model which is realistic and feasible to obtain as a result, for the multispectral Sentinel-2 LULC classification challenge. In the future, pixel neighbouring analysis can be applied to pixel classification to advance the classification of LULC that have a combination of land types for example Shrublands. Deeper layers, on the other hand, advocate learning more criteria, and the network is vulnerable to overfitting. This weakness will be resolved in the future by incorporating an additional robust LULC classification system.

\section{Conflict of interest:}

Funding:

There is no conflict of interest.

Availability of data and material:

There is no availability of data and material.

Code Availability:

There is no code availability.

Author's contribution:

There is no author's contribution.

\section{References}


1. M. GOLIPOUR, H. GHASSEMIAN, F. MIRZAPOUR: Integrating hierarchical segmentation maps with MRF prior for classification of hyperspectral images in a Bayesian framework. IEEE Transactions on Geoscience and remote Sensing, 54(2), 2015, 805-816.

2. A. GHOSH, B.N. SUBUDHI, L. BRUZZONE: Integration of Gibbs Markov random field and Hopfield-type neural networks for unsupervised change detection in remotely sensed multitemporal images. IEEE Transactions on Image Processing, 22(8), (2013), 3087-3096.

3. D. MERA, M. FERNÁNDEZ-DELGADO, J. M. COTOS, J. R. R. VIQUEIRA, S. BARRO: Comparison of a massive and diverse collection of ensembles and other classifiers for oil spill detection in sar satellite images. Neural Computing and Applications, 28(1), (2017), 1101-1117.

4. J. SCHMIDHUBER: Deep learning in neural networks: An overview. Neural networks, 61, (2015), 85117.

5. Y. ZHOU, H. WANG, F. XU, Y. Q. JIN: Polarimetric SAR image classification using deep convolutional neural networks. IEEE Geoscience and Remote Sensing Letters, 13(12), (2016), 19351939.

6. W.SUN, R. WANG: Fully convolutional networks for semantic segmentation of very high resolution remotely sensed images combined with DSM. IEEE Geoscience and Remote Sensing Letters, 15(3), (2018), 474-478.

7. V. ALHASSAN, C. HENRY, S. RAMANNA, C. STORIE: A deep learning framework for landuse/Land cover mapping and analysis using multispectral satellite imagery. Neural Computing and Applications, (2019),1-16.

8. S. PAN, H. GUAN, Y. CHEN, Y. YU, W. N. GONÇALVES, J. M. Junior, J.Li: Landcover classification of multispectral LiDAR data using CNN with optimized hyper-parameters. ISPRS Journal of Photogrammetry and Remote Sensing, 166, (2020), 241-254.

9. X.ZOU, M. CHENG, C. WANG, Y. XIA, J.LI: Tree classification in complex forest point clouds based on deep learning. IEEE Geoscience and Remote Sensing Letters, 14(12), (2017), 2360-2364.

10. L. MA, M. ZHOU, C. LI: Land covers classification based on Random Forest method using features from full-waveform LiDAR data. International Archives of the Photogrammetry, Remote Sensing and Spatial Information Sciences, (2017), 42(2/W7). 
11. J. YIM, J. JU, H. JUNG, J. KIM: Image classification using convolutional neural networks with multistage feature. In Robot Intelligence Technology and Applications 3 (pp. 587-594). Springer, (2015), Cham.

12. J. MEI, B. GAO, D. XU, W. YAO, X. ZHAO, H. ZHAO: Semantic segmentation of 3d lidar data in dynamic scene using semi-supervised learning. IEEE Transactions on Intelligent Transportation Systems, 21(6), (2019), 2496-2509.

13. W. ZHOU: An object-based approach for urban land cover classification: Integrating LiDAR height and intensity data. IEEE Geoscience and Remote Sensing Letters, 10(4), (2013), 928-931.

14. M. A. KADHIM, M. H. ABED: Convolutional Neural Network for Satellite Image Classification. In Asian Conference on Intelligent Information and Database Systems (pp. 165-178). Springer, (2019, April), Cham.

15. A. UNNIKRISHNAN, P, S. K. SOWMYA: Deep AlexNet with reduced number of trainable parameters for satellite image classification. Proc. Comput. Sci. pp. 2018, 931-938.

16. A. SHARMA, X. LIU, X.YANG: Land cover classification from multi-temporal, multi-spectral remotely sensed imagery using patch-based recurrent neural networks. Neural Networks, (2018), 105, 346-355.

17. C. QIU, L. MOU, M. SCHMITT, X. X. ZHU: Local climate zone-based urban land cover classification from multi-seasonal Sentinel-2 images with a recurrent residual network. ISPRS Journal of Imagegrammetry and Remote Sensing, (2019), 154, 151-162.

18. C. SHI, C. M. PUN: Adaptive multi-scale deep neural networks with perceptual loss for panchromatic and multispectral images classification. Information Sciences, 490, (2019), 1-17.

19. T. HE, S.WANG: Multi-spectral remote sensing Land cover classification based on deep learning methods. The Journal of Supercomputing, (2020), 1-15.

20. A. B. GAVADE, V. S. RAJPUROHIT: A hybrid optimization-based deep belief neural network for the classification of vegetation area in multi-spectral satellite image. International Journal of Knowledgebased and Intelligent Engineering Systems, 24(4), (2020), 363-379.

21. J. YUE, W. ZHAO, S. MAO, H. LIU: Spectral-spatial classification of hyperspectral images using deep convolutional neural networks. Remote Sensing Letters, 6(6), (2015), 468-477.

22. B. XU: Improved convolutional neural network in remote sensing image classification. Neural Computing and Applications, (2020),1-12. 
23. H. N. T. THANH, T. M. DOAN, E. TOMPPO, R. E. MCROBERTS: Land use/land cover mapping using multitemporal sentinel-2 imagery and four classification methods-A case study from dak nong, vietnam. Remote Sensing, 12(9), (2020), 1367.

24. M. Z. ALOM, M. HASAN, C. YAKOPCIC, T. M. TAHA, V. K. ASARI: Improved inception-residual convolutional neural network for object recognition. Neural Computing and Applications, 32(1), (2020), 279-293.

25. Y. HE, K. KAVUKCUOGLU, Y. WANG, A. SZLAM, Y. QI: Unsupervised feature learning by deep sparse coding. In Proceedings of the 2014 SIAM international conference on data mining (pp. 902910). (2014, April), Society for Industrial and Applied Mathematics. 